\title{
Standard Model measurements with the ATLAS detector
}

\author{
Samira Hassani ${ }^{1, a}$, on behalf of the ATLAS collaboration \\ ${ }^{1}$ DSM/IRFU (Institut de Recherches sur les Lois Fondamentales de l'Univers), CEA Saclay (Commissariat \\ à l'Energie Atomique et aux Energies Alternatives), Gif-sur-Yvette, France
}

\begin{abstract}
Various Standard Model measurements have been performed in proton-proton collisions at a centre-of-mass energy of $\sqrt{s}=7$ and $8 \mathrm{TeV}$ using the ATLAS detector at the Large Hadron Collider. A review of a selection of the latest results of electroweak measurements, $W / Z$ production in association with jets, jet physics and soft QCD is given. Measurements are in general found to be well described by the Standard Model predictions.
\end{abstract}

\section{Introduction}

The successful operation of the Large Hadron Collider (LHC) during the past three years allowed to explore particle interaction in a new energy regime. Various Standard Model measurements have been performed in proton-proton collisions at a centre-of-mass energy of $\sqrt{s}=7$ and $8 \mathrm{TeV}$ using the ATLAS detector [1] at the LHC. These allow for precision tests of the electroweak dynamics of the Standard Model, but also challenge next-to-next-to-leading-order predictions. Differences between measurements and Standard Model predictions could prove evidence for new phenomena. Recent Quantum Chromo Dynamics (QCD) results highlight the importance of precision QCD measurements for improving state-of-the-art theoretical tools and searching for new physics. A selection of the latest results of electroweak measurements, $W / Z$ production in association with jets, jet physics and soft QCD is reported. These consist of the measurement of the $W W$ cross section, first evidence of the electroweak production of $W^{ \pm} W^{ \pm} j j$, and electroweak $Z j j$ production. Measurements are in general found to be well described by the Standard Model predictions. Measurements of differential production cross sections of a $Z$ boson in association with b-jets, the production cross section of the $W$ boson in association with jets as well as the ratio of the production cross sections for $W$ and $Z$ bosons in association with jets are presented. The measurements are compared to next-to-leading-order perturbative QCD calculations and to predictions from different Monte Carlo (MC) generators implementing leading-order matrix elements supplemented by parton showers. Various inclusive, differential or double differential jet measurements are presented. The dominant experimental uncertainty on these measurements comes from the jet energy scale. Next-to-leading order QCD calculations corrected to account for non-perturbative effects are compared to the measurements. A good agreement between the data and the theoretical predictions based on most of the global parton distribution functions is found over the full kinematic range, covering almost seven orders of magnitude in the measured cross-section values. A measurement of charged-particle distributions sensitive to the properties of

\footnotetext{
ae-mail: samira.hassani@cern.ch
} 
the underlying event is shown for an inclusive sample of events containing a $Z$ boson. These results improve the phenomenological models of the underlying event.

\section{Electroweak Measurements}

The measurement of the $W^{+} W^{-}$production cross section provides an important test of the non-Abelian structure of the Standard Model (SM). The $W^{+} W^{-}$production rates and differential cross sections are sensitive to anomalous $W W \gamma$ and $W W Z$ triple gauge boson couplings (TGCs). Furthermore, nonresonant $W^{+} W^{-}$production is an irreducible background process to Higgs boson studies using the same final state. The dominant production mechanisms are $s$-channel and $t$-channel quark-antiquark annihilation $(q \bar{q} \rightarrow W W)$. In addition, $W W$ events can be produced via gluon fusion through a quark loop, including the non-resonant $(g g \rightarrow W W$ ) and the Higgs $(g g \rightarrow H \rightarrow W W$ ) processes. The total theoretical $W W$ production cross section at $\sqrt{s}=8 \mathrm{TeV}$ is determined to be $58.7_{-2.7}^{+3.0} \mathrm{pb}$. The ATLAS measurement is performed using $20 \mathrm{fb}^{-1}$ of data at $\sqrt{s}=8 \mathrm{TeV}$ in three final states with $e^{ \pm} \mu^{\mp}, e^{+} e^{-}$ and $\mu^{+} \mu^{-}$all accompanied by missing energy. The measured $W^{+} W^{-}$production cross section in the total phase space is $71.4_{-1.2}^{+1.2}\left(\right.$ stat $_{-4.4}^{+5.0}(\mathrm{syst})_{-2.1}^{+2.2}(\mathrm{lumi}) \mathrm{pb}$ [2]. The dilepton invariant mass distribution for the $e \mu$ channel is shown in figure 1(left). The diboson background is based on MC simulations and is normalised to the integrated luminosity. The other background processes $t \bar{t}, W+$ jets and Drell-Yan use data-driven estimations. The $W^{+} W^{-}$signal candidate events are taken from MC simulation and are normalised to the cross section measured in this analysis. The shape of this distribution agrees very well between data and predictions. Figure 1(right) shows the cross sections measured in the individual channels as well as the combined cross section and compares them to the standard model prediction obtained using the CT10 [3] parton density function (PDF). The measured combined cross section differs from the SM prediction computed using CT10 PDF by $2.1 \sigma$ using the standard PDF and scale uncertainties. Various contributions to the total cross section like soft- $W W p_{T}$ resummation $[4,5]$; have been neglected here and might cause an increase of a few percent to the predicted cross section.

The first ATLAS measurement of same electric charge $W^{ \pm} W^{ \pm} j j$ vector boson production processes (VBS) using $20 \mathrm{fb}^{-1}$ of data at $\sqrt{s}=8 \mathrm{TeV}$ was reported [6]. Events with two reconstructed same-charge leptons $\left(e^{ \pm} e^{ \pm}, \mu^{ \pm} \mu^{ \pm}\right.$and $\left.e^{ \pm} \mu^{ \pm}\right)$and two or more jets are analysed. Cross sections are measured in two fiducial phase space regions respectively for the sum of electroweak and strong production (inclusive signal region) and for the electroweak process alone. Dominant backgrounds come from events with three or more leptons where one or more leptons are not reconstructed, asymmetric photon conversion in association with another lepton, and non-prompt production of same sign leptons. To reduce $t \bar{t}$ and $W Z / \gamma^{*}+$ jets background, events in the inclusive signal region are required to have $m_{j j}>500 \mathrm{GeV}$. The VBS signal region in addition requires $\left|\Delta y_{j j}\right|>2.4$. An excess of events over the background expectation is observed in both signal regions and in all three channels providing a first evidence for $W^{ \pm} W^{ \pm} j j$ production and electroweak only $W^{ \pm} W^{ \pm} j j$ production with a significance of $4.5 \sigma$ and $3.6 \sigma$ respectively. Figure 2(left) shows the $\left|\Delta y_{j j}\right|$ distribution after the inclusive signal region selections. The measured production cross sections are found to be in agreement with SM predictions. Additional contributions to $W^{ \pm} W^{ \pm} j j$ production can be expressed in a model-independent way using higher-dimensional operators leading to anomalous triple or quartic gauge boson couplings (aTGCs or aQGCs). The measured cross section in the VBS fiducial region is used to set limits on aQGCs affecting vertices with four interacting $W$ bosons. The expected and observed $95 \%$ confidence intervals derived from the profile likelihood function. The one-dimensional observed limits are found to be $-0.14<\alpha_{4}<0.16$ and $-0.23<\alpha_{5}<0.24$ and are obtained setting $\alpha_{4,5}=0$. These to be compared to the expected limits of $-0.10<\alpha_{4}<0.12$ and $-0.18<\alpha_{5}<0.20$. 

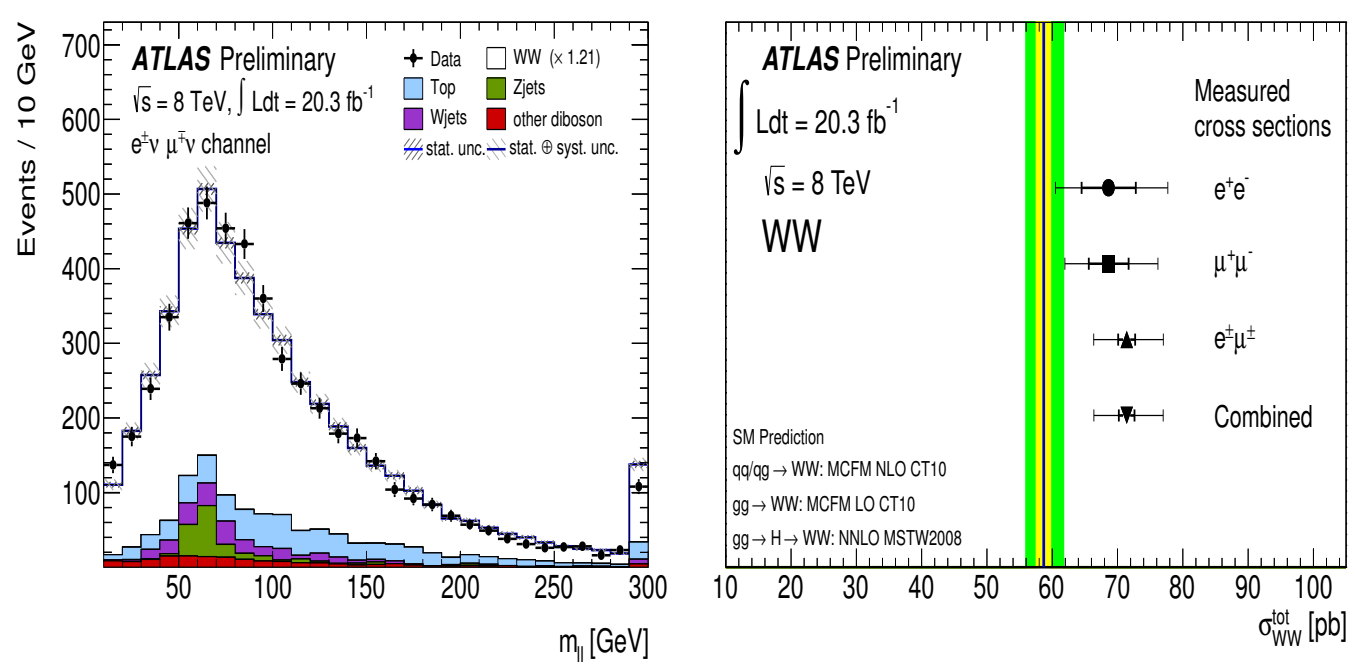

Figure 1. Left: Dilepton invariant mass distribution of the selected $W^{+} W^{-}$candidates in the $e^{ \pm} \mu^{\mp}$ channel. Right: Comparison between predicted $W^{+} W^{-}$production cross section using CT10 PDF and the measured cross section in $e^{ \pm} \mu^{\mp}, e^{+} e^{-}, \mu^{+} \mu^{-}$and combined channels. The yellow and green shaded bands represent the PDF and total theoretical uncertainties respectively. The filled symbols show the measured total cross section with the statistical and total uncertainty [2].
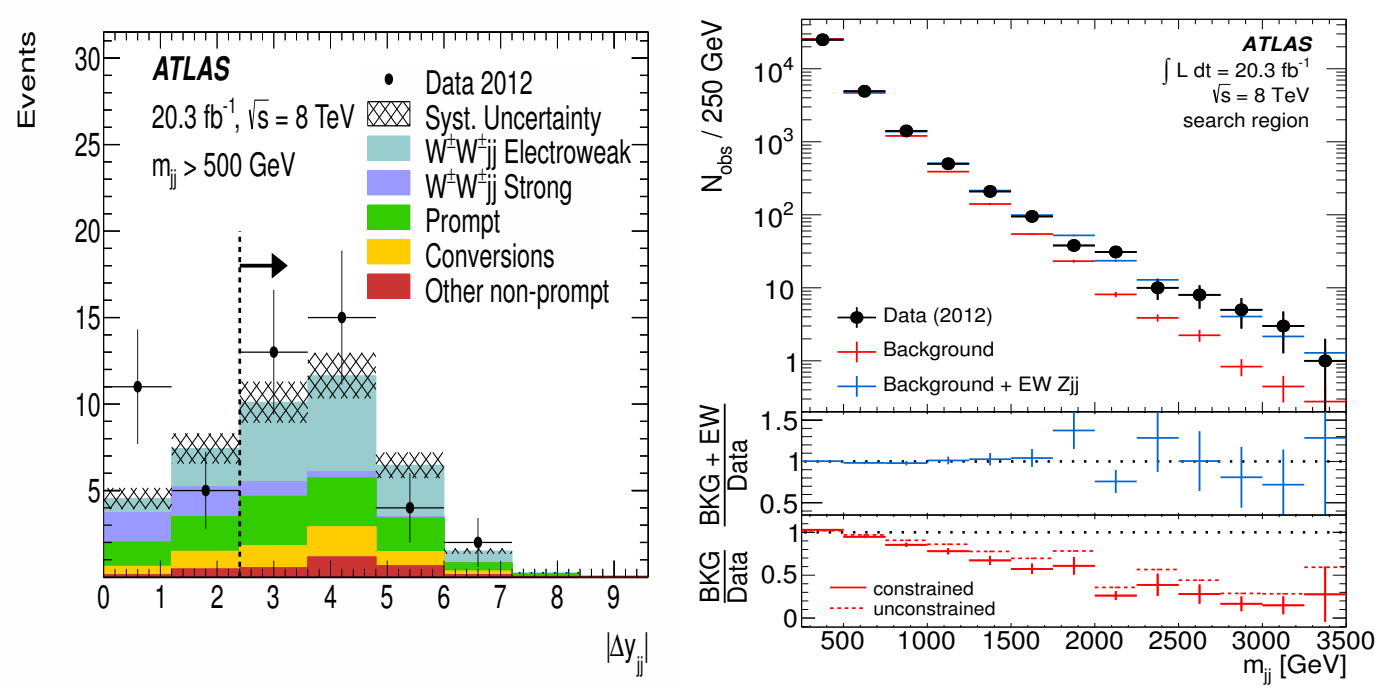

Figure 2. Left: $\left|\Delta y_{j j}\right|$ distribution for events passing all inclusive signal region selections. The contributions from electroweak and strong $W^{ \pm} W^{ \pm} j j$ production are normalized to the SM prediction [6]. Right: The dijet invariant mass distribution in the search region. The signal and background templates are scaled to match the number of events obtained in the fit [7]. 
Measurements of fiducial cross sections for the electroweak production of two jets in association with a $Z$ boson are presented [7]. The measurements are performed using $20 \mathrm{fb}^{-1}$ of data at $\sqrt{s}=$ $8 \mathrm{TeV}$. Electroweak $Z j j$ production include $Z$-boson production via vector boson fusion (VBF), $Z$ boson bremsstrahlung and non-resonant production. The VBF process is of particular interest because of the similarity to the VBF production of a Higgs boson. Candidate events have one opposite-sign same-flavor lepton pair with $81 \mathrm{GeV}<m_{\ell \ell}<100 \mathrm{GeV}$ and the two highest $p_{T}$ jets ("tagging jets") in the event are required to fulfill $m_{j j}>250 \mathrm{GeV}$ and $1 \mathrm{TeV}$, respectively. Additionally, a jet veto on additional jets in the rapidity interval between the two tagging jets is applied. The electroweak component is extracted by a fit to the dijet invariant mass distribution in a fiducial region chosen to enhance the electroweak contribution over the dominant background in which the jets are produced via the strong interaction. Figure 2(right) shows the dijet invariant mass distribution for the electron and muon channels combined. The signal and background templates are normalised to the values obtained from the fit. The electroweak $Z j j$ cross sections measured in the fiducial region for $\left(m_{j j}>250 \mathrm{GeV}\right)$ is found to be $\sigma_{E W}=54.7 \pm 4.6$ (stat) ${ }_{-10.4}^{+9.8}$ (syst) \pm 1.5 (lumi)fb and is in good agreement with the SM theoretical predictions of $46.1 \pm 1.0 \mathrm{fb}$. The background-only hypothesis is rejected with significance above the $5 \sigma$ level.

\section{$3 \mathrm{~W} / \mathrm{Z}$ production in association with jets}

Precise measurements of the production of vector bosons in association with jets are important tests of QCD and provide constraints on background processes to Higgs boson studies and to searches for new physics. Predictions for heavy flavour production typically suffer from larger theoretical uncertainties than those for the more inclusive $Z+$ jets processes, and measurements of $Z$ boson production in association with b-jets can therefore provide important experimental constraints to improve the theoretical description of this process. Two schemes are generally employed in perturbative QCD (pQCD) calculations containing heavy flavour quarks. One is the four-flavour number scheme (4FNS), which only considers parton densities of gluons and of the first two quark generations in the proton. The other is the five-flavour number scheme (5FNS), which allows a b-quark density in the initial state and raises the prospect that measurements of heavy flavour production could constrain the b-quark parton density function (PDF) of the proton.

Differential $Z+b$-jets cross-section measurements have been presented using $4.6 \mathrm{fb}^{-1}$ of data at $\sqrt{s}=7 \mathrm{TeV}$ [8]. The cross-sections for $Z+\geq 1 \mathrm{~b}$-jet and $Z+\geq 2$ b-jets are shown in figure 3 . Next-to-leading-order predictions from MCFM [9] and aMC@NLO [10] generally provide the best overall description of the data. The agreement of the aMC@NLO cross-section prediction with data differs in the $Z+\geq 1$ b-jet and $Z+\geq 2$ b-jets cases, with the former better described by the 5FNS prediction and the latter better described by the 4FNS prediction. Even at NLO, scale uncertainties dominate and currently limit any sensitivity to different PDF sets. Considering only statistical uncertainties, both the 4FNS prediction from ALPGEN [11]+HERWIG [12]+JIMMY [13] and the 5FNS prediction from SHERPA [14] underestimate the data, with ALPGEN+HERWIG+JIMMY being consistently below SHERPA by around $30-40 \%$.

The production cross section of the $W$ boson in association with jets is measured using $4.6 \mathrm{fb}^{-1}$ of data at $\sqrt{s}=7 \mathrm{TeV}$ [15]. Cross sections are shown in figure 4 as a function of the jet multiplicity up to seven jets for electron and muon decay modes of the $W$ boson. The measurements are compared to next-to-leading-order perturbative QCD calculations, resummation calculations and to predictions from different Monte Carlo generators implementing next-to-leading-order and leading-order matrix elements supplemented by parton showers. The data are in good agreement with the predictions from 

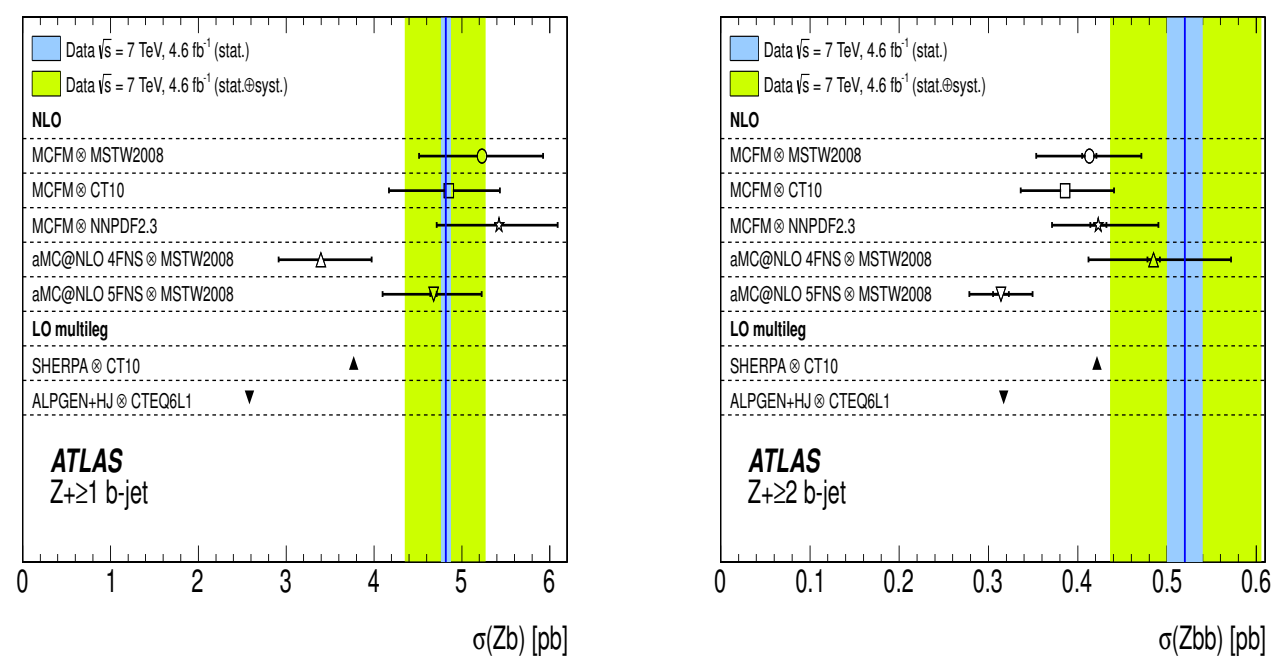

Figure 3. Cross-sections for (left) $Z+\geq 1$ b-jet, and (right) $Z+\geq 2$ b-jets. The measurement is shown as a vertical blue line with the inner blue shaded band showing the corresponding statistical uncertainty and the outer green shaded band showing the sum in quadrature of statistical and systematic uncertainties. Comparison is made to NLO predictions from MCFM interfaced to different PDF sets and aMC@NLO interfaced to the same PDF set in both the 4FNS and 5FNS. The statistical (inner bar) and total (outer bar) uncertainties are shown for these predictions, which are dominated by the theoretical scale uncertainty. Comparisons are also made to LO multi-legged predictions from ALPGEN+HERWIG+JIMMY and SHERPA; in this case the uncertainty bars are statistical only, and smaller than the marker [8].

BlackHat [16]+SHERPA for all jet multiplicities up to five jets. MEPS@NLO [17] also describes the jet multiplicity distribution with a similar level of agreement. The ALPGEN and SHERPA predictions show different trends for jet multiplicities greater than four jets, however both are in agreement to the data within the experimental systematic uncertainties.

The measurement of the ratio of $W+$ jets to $Z+$ jets production cross sections, termed " $R_{\text {jets" }}$, directly probes the difference between the kinematic distributions of the jet system recoiling against the $W$ or $Z$ bosons. In comparison to separate $W+$ jets and $Z+$ jets cross section measurements, the $R_{\text {jets }}$ measurement is a more precise test of pQCD, since some experimental uncertainties and effects from non-perturbative processes, such as hadronization and multi-parton interactions, are greatly reduced in the ratio. Figure 4 shows the $R_{\text {jets }}$ ratio versus the leading-jet $p_{T}$ for $N_{\text {jets }}=1$. At low transverse momentum $\left(p_{T}<200 \mathrm{GeV}\right)$, the $R_{\text {jets }}$ distribution falls as the leading-jet $p_{T}$ increases, indicating that the shapes in $W+$ jets and $Z+$ jets events are different. This is due to the $W$ and $Z$ boson mass difference, which affects the scale of the parton radiation, and the different vector boson polarizations, which affect the kinematics of their decay products. In the small region very close to the minimum value of the jet $p_{T}$ considered in the analysis, where radiative parton shower effects play a major role, all of the predicted shapes exhibit trends different from those in the data, but the ALPGEN predictions still show the best agreement. 

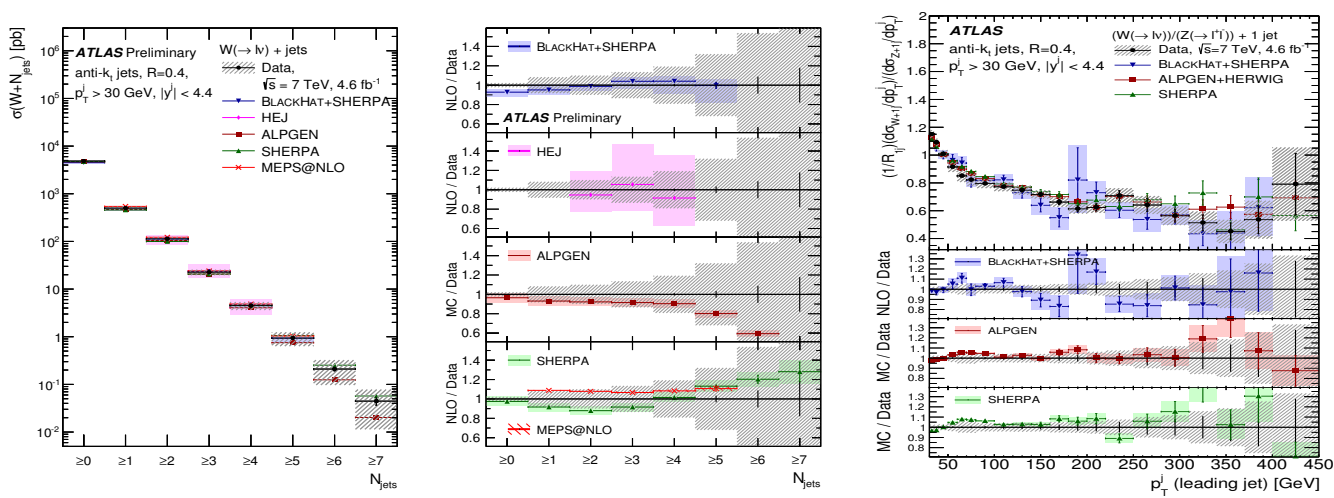

Figure 4. Left : $W+$ jets cross section as a function of the inclusive jet multiplicity. For the data, the statistical uncertainties are shown by the vertical bars, and the combined statistical and systematic uncertainties are shown by the black-hashed regions. Center: the ratios of the predictions to the data. Right : The ratio of $W+$ jets and $Z+$ jets production cross sections, $R_{\text {jets }}$, normalized as described in the text versus the leading-jet transverse momentum, for $N_{\text {jets }}=1$. Ratios of the BlackHat+Sherpa NLO calculation and the Alpgen and Sherpa generators to the data are shown in the lower panels. Vertical error bars show the respective statistical uncertainties [15].

\section{Jet Physics}

The inclusive-jet cross-section has been measured at a centre-of-mass energy of $7 \mathrm{TeV}$ using a data set corresponding to $4.5 \mathrm{fb}^{-1}$ [18]. The inclusive-jet double-differential cross section is shown in figure 5 (left) for jets reconstructed using the anti- $k_{t}$ algorithm with a radius parameters of $R=0.4$. The measurement extends over jet transverse momenta from $100 \mathrm{GeV}$ to $2 \mathrm{TeV}$ in the rapidity region of $|y|<3$. The NLO pQCD predictions calculated with NLOJET++ [19] using the CT10 PDF set with corrections for non-perturbative effects and electroweak effects applied are compared to the measurement. Figure 5 shows that the NLO pQCD predictions follow the measured cross sections which range over eight orders of their magnitude in the six rapidity bins. A quantitative comparison of the measured cross sections to the QCD calculations using several sets of parton distribution functions is performed. A next-to-leading-order QCD analysis shows an impact of the measured jet cross section on the determination of the gluon parton distribution function.

Double-differential three-jet production cross sections have also been measured at $7 \mathrm{TeV}$ using a data set corresponding to $4.5 \mathrm{fb}^{-1}$ [20]. The measurement is presented in figure 5(right) as functions of the three-jet invariant mass $\left(m_{j j j}\right)$ and the sum of absolute rapidity separations between the three leading jets $\left(\left|Y^{*}\right|\right)$. Invariant masses extending up to $5 \mathrm{TeV}$ are reached for $8<\left|Y^{*}\right|<10$. The total experimental uncertainty in these measurements is dominated by the jet energy scale calibration uncertainty. The cross section decreases rapidly as a function of the three-jet invariant mass. The QCD calculations by NLOJET++ using the CT10 PDF set corrected for non-perturbative effects are compared to the measured cross sections. Good agreement between the data and the theoretical predictions is found over the full kinematic range, covering almost seven orders of magnitude in the measured cross section values.

Additional jet activity in dijet events is measured using data at a centre-of-mass energy of $7 \mathrm{TeV}$ [21] , for jets reconstructed using the anti- $k_{t}$ algorithm with $R=0.6$. This is done using vari- 

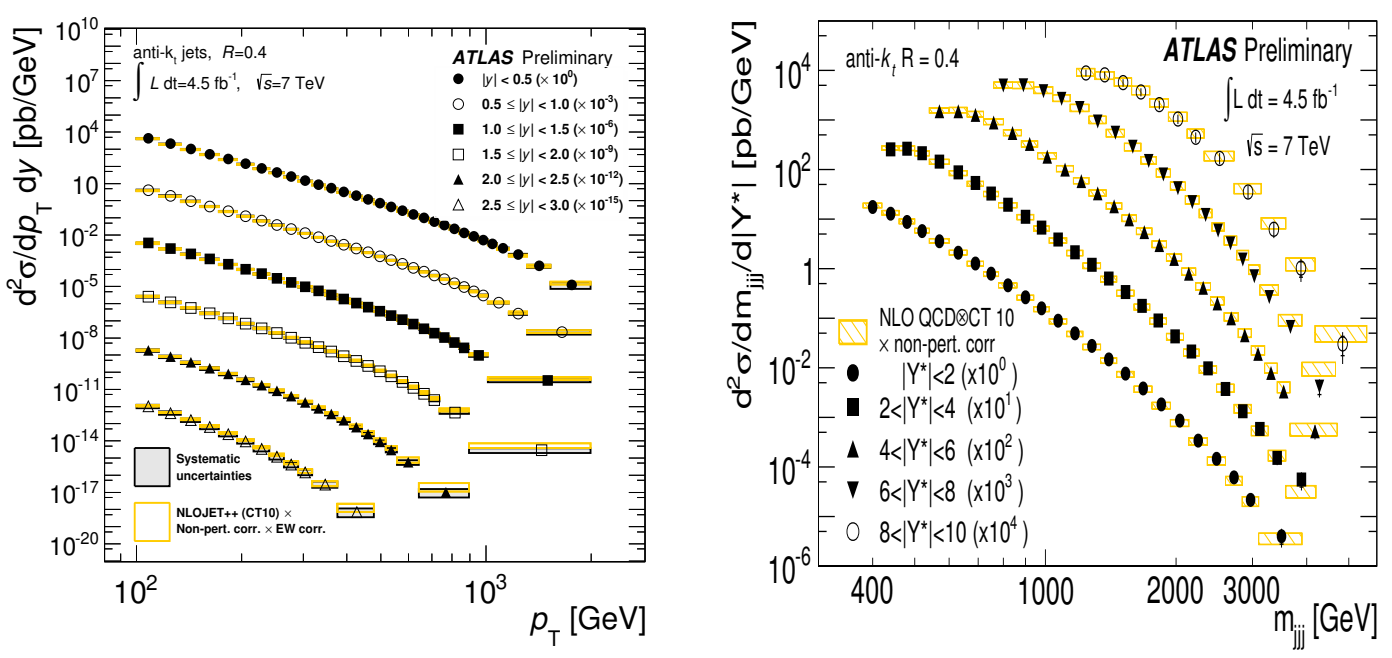

Figure 5. Left: Inclusive-jet double-differential cross sections as a function of the jet $p_{T}$ in bins of rapidity [18]. Right: Three-jet double-differential cross section as a function of $m_{j j j}$, binned in $\left|Y^{*}\right|$. The results are shown for jets identified using the anti- $k_{t}$ algorithm with $R=0.4$. For convenience, the cross sections are multiplied by the factors indicated in the legend [20].

ables such as the fraction of dijet events without an additional jet in the rapidity interval $(\Delta y)$ bounded by the dijet subsystem and correlations between the azimuthal angles of the dijets. These variables probe differences in the approach to resummation of large logarithms when performing QCD calculations. A particularly interesting observable is the gap fraction, $f\left(Q_{0}\right)$, defined as $f\left(Q_{0}\right)=\frac{\sigma_{j j}\left(Q_{0}\right)}{\sigma_{j j}}$ where $\sigma_{j j}$ is the inclusive dijet cross section and $\sigma_{j j}\left(Q_{0}\right)$ is the cross section for dijet production in the absence of jets with transverse momentum greater than $Q_{0}$ in the rapidity interval bounded by the dijet system. The variable $Q_{0}=20 \mathrm{GeV}$ is referred to the veto scale. Figure 6 (left) shows the measured gap fraction as function of $\Delta y$. The data are compared to POWHEG [22], interfaced to the PYTHIA 8 [23] and HERWIG parton shower generators, as well as to HEJ [24] with and without interfacing it to the ARIADNE [25] parton shower generator. None of the theoretical predictions agree with the data across the full phase-space considered; however, POWHEG+PYTHIA 8 and HEJ+ARIADNE are found to provide the best agreement with the data.

\section{Soft QCD}

In order to perform precise Standard Model measurements or to search for new physics phenomena at hadron colliders, it is important to have a good understanding of not only the short-distance hard scattering process, but also of the accompanying activity collectively termed the underlying event (UE). Measurement of distributions sensitive to the UE, performed with the ATLAS detector are presented using a data set corresponding to $4.6 \mathrm{fb}^{-1}$ at centre-of-mass energy of $7 \mathrm{TeV}$ [26]. Events with a $Z$-boson candidate decaying into an electron or muon pair were selected, and observables constructed from the final state charged particles (after excluding the lepton pair) were studied as a function of the transverse momentum of the $Z$-boson candidate $\left(p_{T}^{Z}\right)$. The transverse and toward 

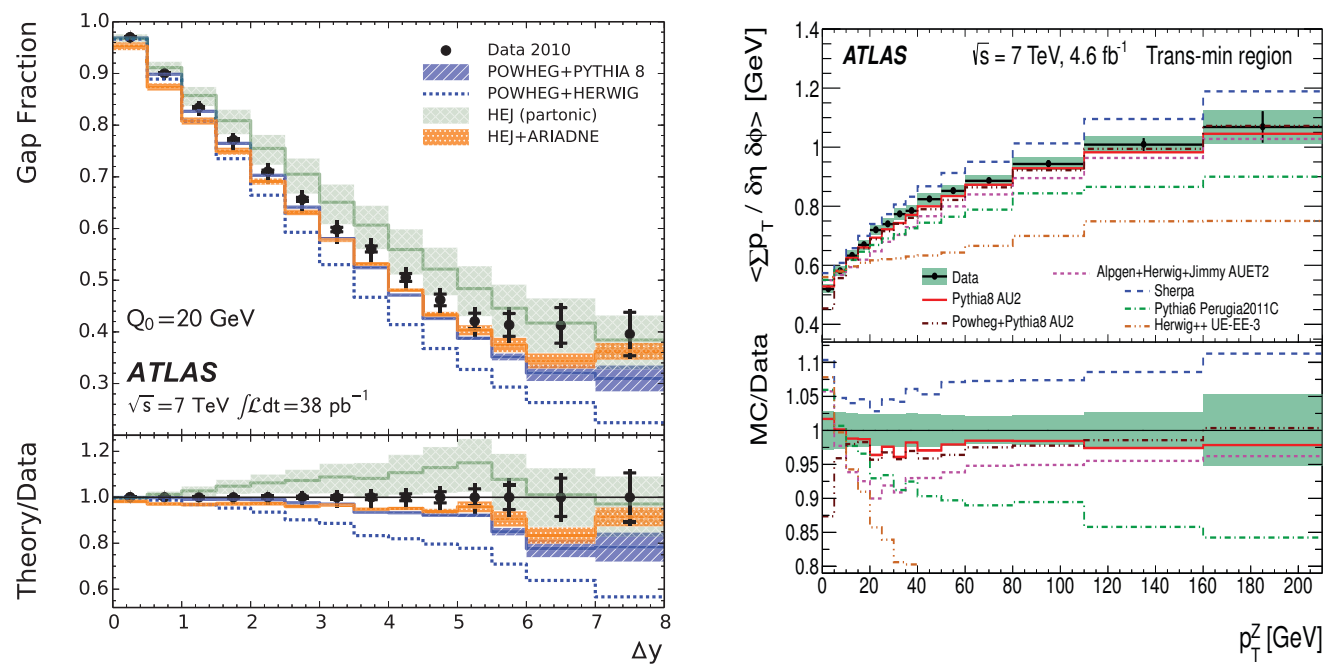

Figure 6. Left: The measured gap fraction (black dots) as a function of $\Delta y$ [21]. Right: Comparison of data and $\mathrm{MC}$ predictions for charged particle scalar $\sum p_{T}$ density profile, $\left\langle\sum p_{T} / \delta \eta \delta \phi>\right.$, as a function of $Z$-boson transverse momentum $p_{T}^{Z}[26]$.

regions with respect to the reconstructed $Z$-boson are most sensitive to the underlying event. The observable $\sum p_{T} / \delta \eta \delta \phi$ defined as scalar $p_{T}$ sum of stable charged particles per unit $\eta-\phi$, is shown in figure 6(right) as a function of $p_{T}^{Z}$ and is compared with the predictions of various MC models using several tunes of commonly used underlying event models. MC model predictions qualitatively describe well the data, but with some significant discrepancies, providing precise information sensitive to the choices of parameters used in the various underlying-event models. Careful tuning of these parameters in the future may improve the description of the data by the different models in future LHC measurements and studies. The study of such variables in Z-boson events provides a probe of the underlying event which is complementary to that from purely hadronic events. A comparison between them shows similar underlying event activity for the transverse region.

\section{Summary}

The data collected during the first phase of operation at the LHC have been used to extract precise measurements of many Standard Model processes. Excellent agreement is found for all the processes considered within the uncertainties. This is a remarkable achievement of the Standard Model and the underlying theoretical concepts, including QCD and factorization. In 2015 the LHC will resume operation near its design energy of $14 \mathrm{TeV}$, and will continue the quest for new physics through direct searches for new particles and through precision measurements of Standard Model processes and parameters.

\section{Acknowledgements}

This report was supported by the European Research Council, Grant Agreement $N^{\circ} 279170$ DIBOSON. 


\section{References}

[1] ATLAS Collaboration, JINST, 3 (2008) S08003.

[2] ATLAS Collaboration, ATLAS-CONF-2014-033, http://cds.cern.ch/record/1728248.

[3] P. M. Nadolsky et al., Phys.Rev.D, 78 (2008) 013004.

[4] Prerit Jaiswal, Takemichi Okui, arXiv:1407.4537.

[5] Patrick Meade, Harikrishnan Ramani, Mao Zeng, arXiv:1407.4481.

[6] ATLAS Collaboration, accepted by PRL, arXiv:1405.6241(2014).

[7] ATLAS Collaboration, JHEP, 04 (2014) 031.

[8] ATLAS Collaboration, submitted to JHEP, arXiv:1407.3643.

[9] J. Campbell et al., arXiv:1007.3492.

[10] J. Alwall et al., arXiv:1405.0301.

[11] M. L. Mangano et al., JHEP 0307 (2003) 001, arXiv:hep-ph/0206293.

[12] G. Corcella et al., JHEP 0101 (2001) 010, arXiv:hep-ph/0011363.

[13] J. M. Butterworth et al., Z. Phys. C 72 (1996) 637, arXiv:hep-ph/9601371.

[14] T. Gleisberg et al., JHEP 0902 (2009) 007.

[15] P. Nason, JHEP 11040 (2004).

[16] C. Berger et al. , Phys.Rev.D78 (2008) 036003, arXiv:0803.4180.

[17] T. Gleisberg et al., JHEP 0902 (2009) 007, arXiv:0811.4622.

[18] ATLAS Collaboration, https://atlas.web.cern.ch/Atlas/GROUPS/PHYSICS/PAPERS/STDM2013-11/

[19] Z. Nagy, Phys. Rev. D 68 (2003) 094002, [hep-ph/0307268].

[20] ATLAS Collaboration, ATLAS-CONF-2014-045, https://cds.cern.ch/record/1741019/

[21] ATLAS Collaboration, https://atlas.web.cern.ch/Atlas/GROUPS/PHYSICS/PAPERS/STDM2012-17/

[22] S. Alioli et al., JHEP 07 (2008) 060, arXiv:0805.4802.

[23] T. Sjostrand et al., Comput. Phys. Commun. 178 (2008) 852-867, arXiv:0710.3820.

[24] J. R. Andersen et al. , Nucl. Phys. Proc. Suppl. 205-206 (2010) 205-210, arXiv:1007.4449.

J. R. Andersen and J. M. Smillie, JHEP 1106 (2011) 010, arXiv:1101.5394.

[25] L. Lonnblad, Comput. Phys. Commun. 71 (1992) 15-31.

[26] ATLAS Collaboration, Submitted to EPJC, arXiv:1409.3433. 